九州大学学術情報リポジトリ

Kyushu University Institutional Repository

\title{
Sporenpaläontologische Untersuchungen der Hioki-Schichtengruppe von Waku und Kiwado
}

Takahashi, Kiyoshi

Faculty of Science, Kyushu University

https://doi.org/10.5109/1543607

出版情報：九州大學理學部紀要：Series D, Geology. 14 (2)，pp.143-157，1963-03-01. Faculty of Science, Kyushu University バージョン：

権利関係 : 
Mem. Fac. Sci., Kyshu Univ., Ser. D, Geology, Vol. XIV, No. 2, pp. 143-157, text-figs. 1-5, tables 1-2, Plates 20-22, March 1, 1963.

\title{
Sporenpaläontologische Untersuchungen \\ der Hioki-Schichtengruppe von Waku und Kiwado*
}

Von

Kiyoshi TAKAHASHI

\begin{abstract}
Zusammenfassung
Drei Proben aus der Hioki-Schichtengruppe bei Waku und Kiwado wurden pollenanalytisch untersucht. In diesem Pollenspektrum tritt Alnus-Pollen mit dem höchsten Prozentsatz auf. Ungeflügelter Koniferen-Pollen (pseudodubius.Typus), andere Vieleckpollen, Liquidambar-oder Tilia-Pollen sind weniger zahlreich, Cupuliferae-Pollen der Tricolpopoll.-Form und Monocolpopoll.-Pollen sehr selten. Diese Eigentümlichkeiten des Pollenspektrums sind denen des Sasebo-Pollenbildes gleich.

Neue Formen, Gleicheniidites triangularis n. sp., Btulaepoll. rarus n. sp., Subtriporopoll. consimilis n. sp., Polyporopoll. sp., Porocolpopoll. sp., Tricolporopoll. sp. u. a., wurden eingehend beschrieben.
\end{abstract}

\section{Einleitung}

Bezüglich der tertiären Formationen in der Yuya-wan- und Kottoi-Gegend gibt es frühere Untersuchungen von T. Ogura $(1918,1919) \mathrm{u}$. a. In den letzteren Jahren haben S. Imamura, K. WAdA und K. OKамото sie weiter eingehend untersucht. Nach K. Окамото kann die Hioki-Schichtengruppe mit der Ashiya-Schichtengruppe verglichen werden.

Der Verfasser (1961) hat zahlreiche Sporen und Pollen des westjapanischen Alttertiärs und Untermiozäns beschrieben und eine Pollenstratigraphie aufgestellt. Danach hat er über die Sporen und Pollen des Sakamizu-Schichten der Ashiya-Schichtengruppe berichtet und weiter bewiesen, daß die Ashiya-Schichtengruppe nicht zum oberen Onga-Pollenbild, sondern zum Sasebo-Pollenbild gehört. Deshalb gibt es pollenstratigraphisch für die Hioki-Schichtengruppe zwei Möglichkeiten des Vergleiches, nämlich den Vergleich mit der Ashiya- oder Sasebo-Schichtengruppe.

Wertvolle Hinweise verdankt der Verfasser Herrn Prof. Dr. Ryuzo Toriyama sowie Herrn Assistent-Prof. Dr. Tsugio Shuto. Ebenso muß er auch Herrn Prof. Dr. Hermann WeYland, Wuppertal-Elberfeld, für einige Hinweise und die Korrektur seines Schriftsatzes und Herrn Kazuo Окамото an der Universität Hiroshima, der ihm seine wertvolle Meinung mitteilte, danken.

* Received October 15, 1962.

Diese Arbeit wurde am 30. September 1962 in der 82. regelmäßigen Tagung der japanischen paläontologischẹn Gęsellsşchaft in Tokyo veröffentlicht. 


\section{Bisherige geologische und paläontologische Untersuchungen der tertiären Formationen von Kottoi und Kiwado}

Tertiäre Schichten in den betreffenden Gegenden hat früher T. OguRA (1918, 1919) untersucht. Er hat sie auf Grund von Lithothamnium ramosissium Reuss von Shiraishi als Miozän angesehen.

K. WADA, S. Imamura und A. Hase (1951) haben tertiäre Ablagerungen in dem östlichen Bezirk von Yuya-wan in eine untere und obere Schichtengruppe eingeteilt. Sie haben weiter die untere Schichtengruppe in die Kiwado-, Hekinaka- und IgamiSchichten unterteilt und aus den Kiwado-Schichten folgende Molluskenreste genannt: Glycymeris cisshuensis MaKIYAMA, Crassatellites yabei NAGAO, Pitaria ashiyaensis Nagao, Chlamys ashiyaensis NAgAo, Acila mirabilis var. ashiyaensis Nagao u. a. Aus den Hekinaka-Schichten wurden folgende Spezies erwähnt: Tellina maxima NAGAO, Crenella subformicata NAgAo, Venericardia subnipponica NAGAO, Glycymeris cisshuensis Makiyama u. a. Diese Fauna ist der Ashiya-Fauna sehr ähnlich. Die obere Schichtengruppe wurde Yuya-wan-Schichtengruppe genannt. Ihr geologisches Alter ist unteres Miozän.

K. WADA und S. Imamura (1952) haben über die alttertiären Schichten im nordwestlichen Bezirk von Toyoura-gun, die mit einem Teil der oberen und unteren Kiwado-Schichten im östlichen Bezirk von Yuya-wan verglichen werden können, berichtet. Diese Schichten enthalten Balanus sp., Ostrea sp., Venericardia subnipponica NagAo, Ostrea sakitoensis Nagao, Tellina maxima NAgAo, Nuculana sp., Glycymeris cisshuensis MakiYama, Turritella cf. karatsuensis Nagao, Dentalium sp. u. a.

Auf der Ashiya-Schichtengruppe haben S. IMAmuRA und K. WADA (1956) die Hishikai-Schichtengruppe konkordant lagernd neu festgestellt und in dieser Schichtengruppe Daibo-Flora gefunden. Sie meinen, daß die Daibo-Flora älter als die AniaiFlora sei. Die Daibo-Flora besteht aus folgenden Spezies: Acer subpictum SAP., Acer hishikaiensis HuzıokA, Cf. Acer yubariensis HuzıoKa, Carpinus grandis Ung., Cinnamomum sp., Fagus Antipofi HeEr, Koelreuteria cf. eointegrifolia ENDo, Liquidambar formosana HANCE, Liquidambar elegantifolia HuziokA, Magnolia sp., Platanus cf. aceroides GoEPP, Populus cf. balsamoides GoEPP., Ulmus longifolia GoEPP., Zelkova ungeri (ETT. ) Kovats.

S. IMAMURA (1958) hat auch Noda-Flora aus den Oouchiyama-Schichten mitgeteilt. Er hat geäußert, daß ihr geologisches Alter Oligozän sei. Die Noda-Flora besteht aus den folgenden Spezies: Populus Zaddachi HeEr, Quercus cf. groenlandica Heer, Quercus Lyelli Heer, Broussonetia cf. Imaii ENDo, Cercidiphyllum eojaponica Endo, Platanus aceroides GoEPP., Acer arcticum HeER, Celastrophyllum cf. crenatum HeER, Rhamnus cf. Rossmässleri UNG., Viburnum Nordenskiöldi HeER, Betula Brongniarti EтT., Fagus Antipofi HeER.

S. Imamura und K. Окамото (1959) haben die Hishikai-Schichtengruppe widerrufen, nur die Hitomaru-Schichten beibehalten und die Igami-Schichten zu der Yuyawan-Schichtengruppe gestellt und sie haben die Ashiya-Schichtengruppe in die Sakaigawa-, Oouchiyama- und Taoyama-Schichten geteilt.

K. Окамото (1960) hat Foraminiferenreste aus der Ashiya- und Yuya-wan-Schichtengruppe als vorläufige Mitteilung veröffentlicht. $\operatorname{Er}$ hat die erstere mit der Ashiya-Schichtengruppe von Nordkyushu und die letztere mit den Kawai- und KuriSchichten (mittleres Miozän) von Sanin verglichen. 
Tabelle 1. Stratigraphie der tertïren Schichten in der Yuya-wan Gegend. (nach K Окамото)

\begin{tabular}{|c|c|c|}
\hline Mittelmioz:n & \multicolumn{2}{|c|}{ Yuya-wan-Schichtengruppe } \\
\hline \multirow{3}{*}{ Oberoligozän-Untermiozän } & Hitomaru-Schichten \\
& Hioki-Schichtengruppe & Taoyama-Schichten \\
\hline & Oouchiyama-Schichten \\
\hline & Basisgesteine & Sakaigawa-Schichten \\
\hline
\end{tabular}

K. Okamoto und S. Imamura (1962) haben die Ashiya-Schichtengruppe in der Yuya-wan Gegend in die Hioki-Schichtengruppe umgestellt und betont, daß sie mit der Ashiya-Schichtengruppe von Nordkyushu verglichen werden kann.

\section{Fundorte der Proben}

Bei dieser Arbeit wurden drei Proben untersucht. Die geographischen Fundstätten der Proben werden in der Abbildung 1 dargestellt. In Waku hat der Verfasser zwei Proben der humosen Schiefertone an der Meeresküste gesammelt. Der humose Schieferton von Waku (A) ist schwarz und sehr dünn, nur etwa $4 \mathrm{~cm}$ mächtig (s. Abb. 2). Der humose Schieferton von Waku (B) liegt unter etwa 4 bis $5 \mathrm{~m}$ des oberen Schifertons Waku (A). Er ist dunkelbraun und dünn, etwa $8 \mathrm{~cm}$ mächtig (s. Abb. 3). In Kiwado hat der Verfasser eine Braunkohlenprobe an der Meeresküste gesam-

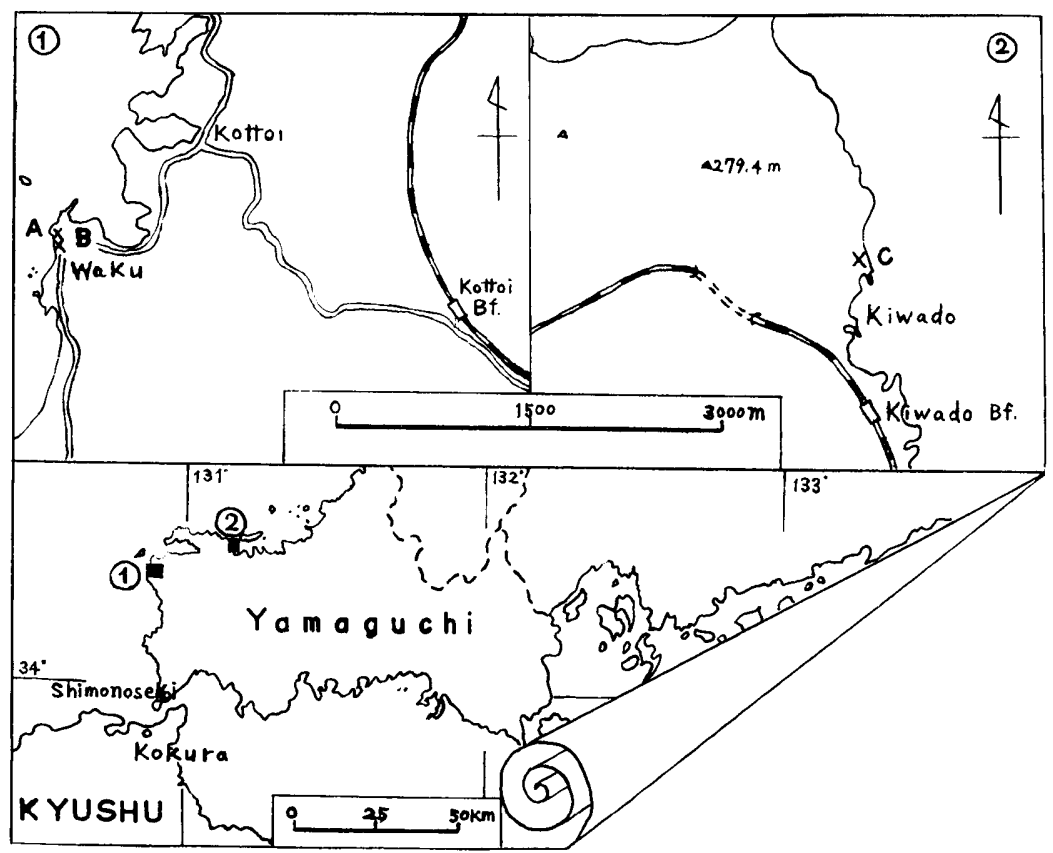

Abb. 1. Fundorte der drei Proben von Waku und Kiwado. 
melt. Das Braunkohlenflöz ist $30 \mathrm{~cm}$ mächtig (s. Abb. $1 \mathrm{u} .4$ ). gehören zu den Sakaigawa-Schichten von K. Окамото.

Diese drei Proben

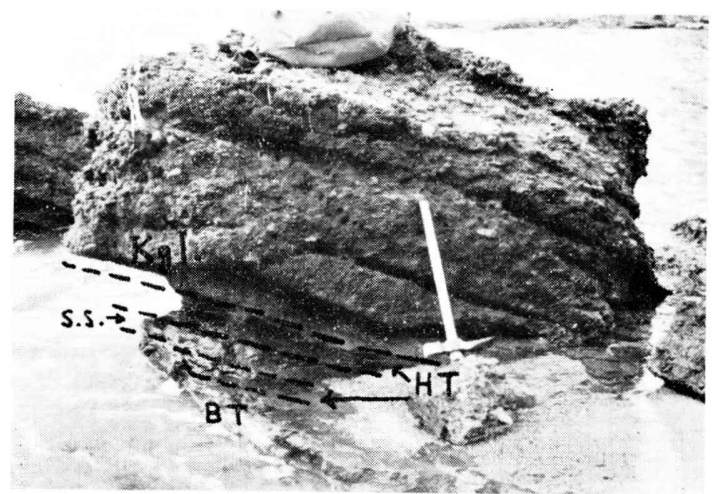

Abb. 2. Fundort der Probe Waku (A).

Kgl. : Konglomerat HT: Humoser Schieferton S. S. : Sandstein BT: Blauer Schieferton

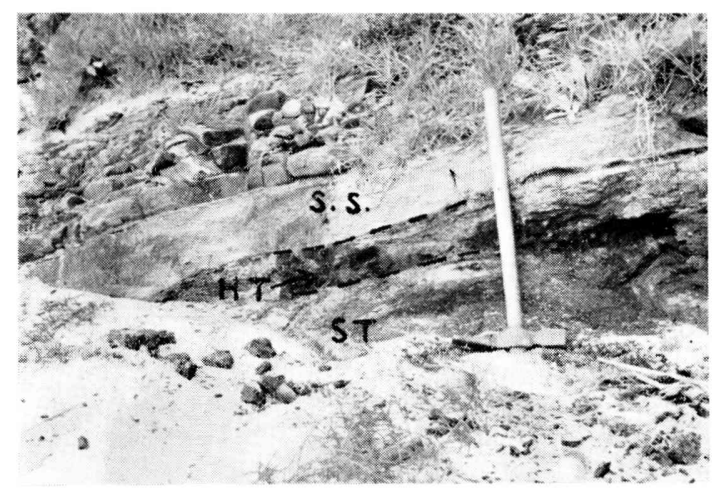

Abb. 3. Fundort der Probe Waku (B).

S. S. : Sandstein HT: Humoser Schieferton ST: Schieferton

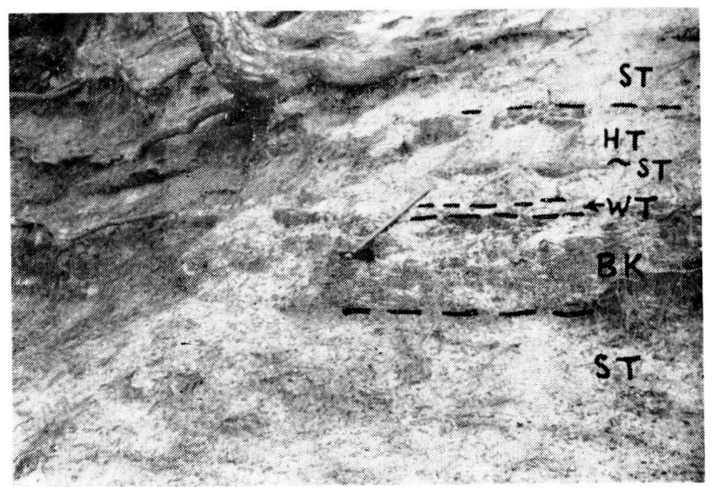

Abb. 4. Fundort des Braunkohlenflözes in Kiwado (C). ST: Schieferton HT: Humoser Schieferton WT: Weißtuff(?) BK: Braunkohle 


\section{Sporen und Pollen}

Die Proben von Waku wurden im Eisenmörser zerkleinert und mit dem Schulzeschen Gemisch $\left(\mathrm{HNO}_{3}+\mathrm{KClO}_{3}\right)$ und Fluorwasserstoffsäure (HF) behandelt.

Der Verfasser hat 1961 einige paläogene und untermiozäne Pollenbilder von Westjapan dargestellt. Dabei hat er eine große Veränderung der Pflanzenwelt zwischen dem oberen Onga- und Sasebo-Pollenbild erkannt. Vor kurzem hat er aber ein dem Sasebo-Pollenbild gleiches Pollenspektrum in dem Schieferton der Sakamizu-Schichten der Ashiya-Schichtengruppe gefunden. Diese Tatsache ist für die tertiäre Pollenstratigraphie von Japan sehr wichtig und auch für die betreffende Pollengruppe.

Hier in der Hioki-Schichtengruppe von Waku und Kiwado kann man CupuliferenPollen der Tricolpaten-Form, die im allgemeinen im Alttertiär häufig sind, nur sehr selten finden. Alnipoll. eminens (TAKaHASHI) tritt mit höchstem Prozentsatz (43.5 bis $83.5 \%$ ) auf. Außer dem Alnus-Pollen sind Polyporopoll. grandis TAKahashi, Polyporopoll. similaris TAKAHASHI und Ulmipoll. undulosus WolfF mit verhältnismäßig höherem Prozentsatz vorhanden. Ungeflügelter Koniferen-Pollen (pseudodubiusTypus) kommt mit 5 bis $7 \%$ vor. Palmae-Pollen ist sehr selten. Von Monocolpopoll. kyushuensis TAKahashi, Monocolpopoll. pflugii TAKahashi und ? Monocolpopoll. intrabaculatus TAKahashi wurde nur ein sehr geringer Prozentsatz gefunden. Periporopoll. asiaticus TAKaHASH und Tiliaepoll. tiliaceus (TAKaHASHI) kommen mit höherem Prozentsatz vor. Corrugatispor. haradae TAKAHASHI und Gleicheniidites triangularis n. sp., die früher in der Sasebo-Schichtengruppe gefunden wurden, wurden auch hier beobachtet. Subtriporopoll. consimilis n. sp., Polyporopoll. sp., Porocolpopoll. sp., Tricolporopoll. hitomaruensis TAKahashr, Tricolporopoll. sp. (mit Netzskulptur) u. a. scheinen lokal beschränkt aufzutreten.

Pollen und Sporen aus den drei Proben verteilen sich in folgender Weise: Waku (A):

1) Ungeflügelter Koniferen-Pollen $=7 \%$

Inaperturopoll. pseudodubius TAKAHASHI …..........................................7\%

2) Geflügelter Koniferen-Pollen $=1 \%$

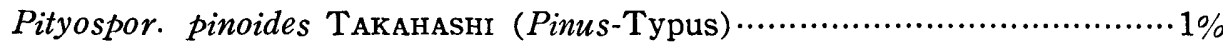

3) Dreieck- und Vieleckpollen $=74.5 \%$

Betulaepoll. rarus TAKAHASHI n. sp. …..................................... 0.5\%

Triporopoll. shimensis TAKaHASHI (cf. Betulaceae) ......................... 0.5\%

Momipites constatus (TAKaHASHI) (Betulaceae) .............................. 0.5\%

Subtriporopoll. kyushuensis TAKaHaSHI (Carya ?) ............................. 0.5\%

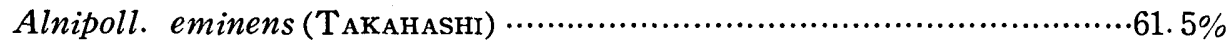

Polyporopoll. grandis TaKaHashi (Ulmaceae) …............................. 4.5\%

Polyporopoll. similaris Takahashi (Juglandaceae ?) …....................... 1.5\%

Ulmipoll. undulosus WoLfF (Ulmus u. Zelkova) …............................. $3 \%$

Tiliaepoll. tiliaceus (TAKAHASHI) ….......................................... $2 \%$

4) Tricolpaten-Pollen $=4 \%$

? Tricolpopoll. ditis Takahashi (Cupuliferae) ….............................. 0.5\%

Tricolpopoll. vulgaris TaKahashi (cf. Cupuliferae) …........................ 0.5\%

Tricolpopoll. meinohamensis TaKaHASHI rotundus TaKaHaSHI............. $0.5 \%$

Tricolpopoll. meinohamensis TAKAHASHI meinohamensis

TAKaHASHI (Cupuliferae ?) ............. $0.5 \%$

Tricolpopoll. chikushiensis TAKaHaSHI grandiformis TaKaHASHI ……....... $5 \%$ 

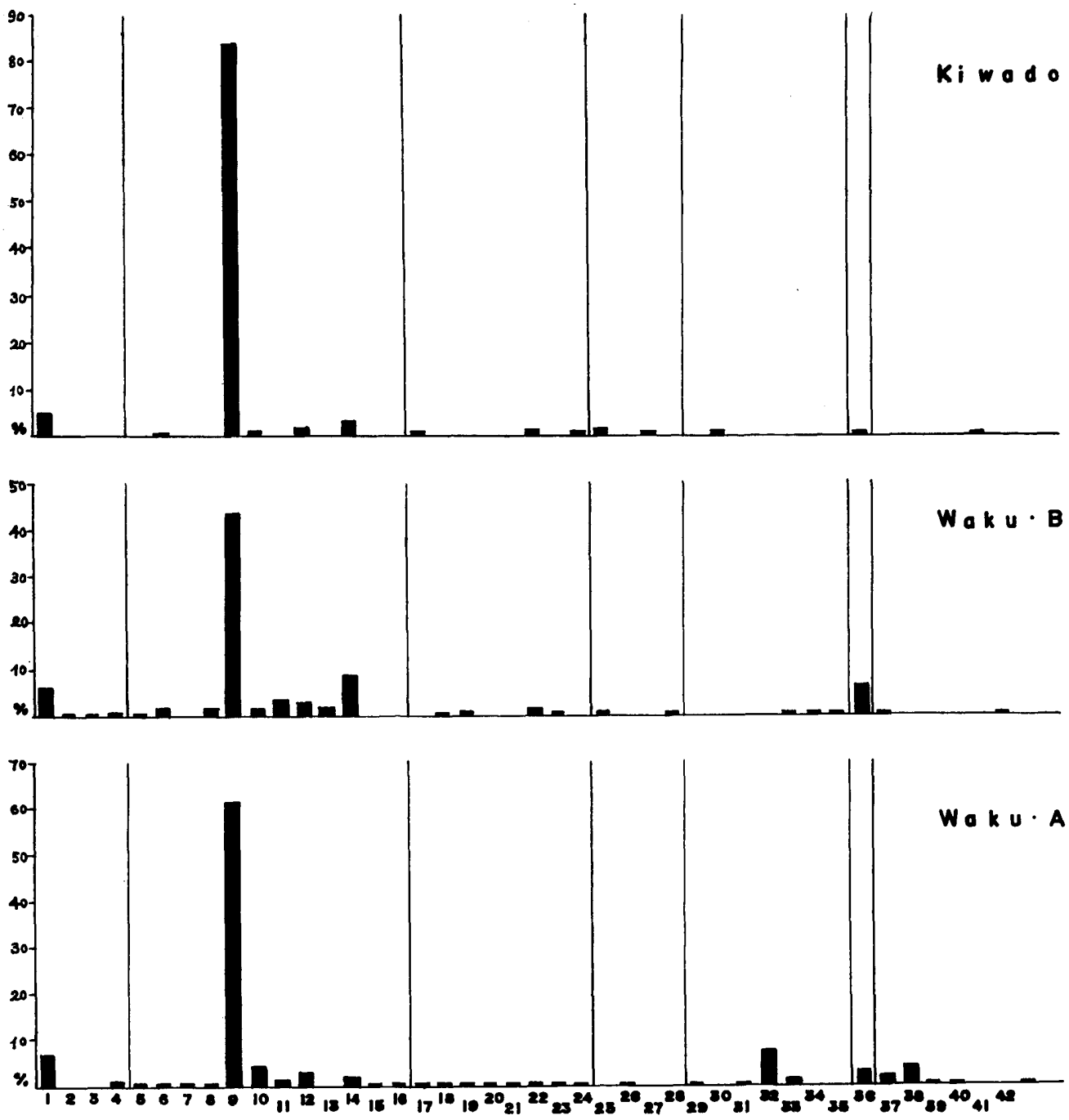

Abb. 5. Pollendiagramm der Hioki-Schichtengruppe.

1: Inaperturopoll. psudodubius TAKaHASH

2: Sequoiapoll. ligularis (ТАKAHASHI)

3: Pityospor. orientalis TakaHashr

4: Pityospor. pinoides TaKaHashi

5: Betulaepoll. rarus $\mathrm{T}_{\mathrm{AK} A \mathrm{HASHI}}$

6: Triporopoll. shimensis TAKAHASH

7. Momipites constatus ( $\left.\mathrm{T}_{\mathrm{AKAHASHI}}\right)$

8: Subtriporopoll. kyushuensis TaKaHashi

9: Alnipoll. eminens (TAKaHashI)

10: Polyporopoll. grandis TAKAHASH

11: Polyporopoll. similaris TaKaHashi

12: Ulmipoll. undulosus WoLFF

13: Subtriporopoll. consimilis TAKAHaSH

14: Tiliaepoll. tiliaceus (T $\mathrm{\text {AKAHASHI) }}$

15: Polyporopoll. sp.
16: Porocolpopoll. sp.

17: ? Tricolpopoll. ditis $\mathrm{T}_{\mathrm{AK} A H A S H I}$

18: Tricolpopoll. vulgaris $\mathrm{T}_{\mathrm{AKAHASHI}}$ (Waku A); Tricolpopoll. umiensis TAKaHashI (Waku B)

19: Tricolpopoll. meinohamensis Takahashi rotundus TAKaHASHI

20: Tricolpopoll. meinohamensis TAKaHASH meinohamensis $\mathrm{T}_{\mathrm{AK}}$.

21: Tricolpopoll. chikushiensis $\mathrm{T}_{\mathrm{AK}}$. grandiformis $\mathrm{T}_{\mathrm{AK}}$.

22: Tricolpopoll. reticulatus TAKaHaSHI

23: Tricolpopoll. microreticulatus TAKAHASH

24: Tricolpopoll. striatellus $\mathrm{T}_{\mathrm{AKAH}} \mathrm{TSHI}_{\mathrm{I}}$

25: Monocolpopoll. kyushuensis $\mathrm{T}_{\mathrm{AKAHASHI}}$

26: ?Monocolpopoll. intrabaculatus TAKAHASHI 
Tricolpopoll. reticulatus TaKaHASHI (Salix, Platanus u. a.) )................... $0.5 \%$

Tricolpopoll. microreticulatus TaKahashi (Salix, Platanus u. a.) )........ $0.5 \%$

Tricolpopoll. striatellus TAKAHASHI .......................................... $0.5 \%$

5) Monocolpaten-Pollen $=0.5 \%$

? Monocolpopoll. intrabaculatus TaKaHaSHI (Palmae) …...................... $0.5 \%$

6) Tricolporaten -Pollen $=2.5 \%$

Tricolporopoll. incertus TAKaHASHI …............................................ 0.5\%

Tricolporopoll. nagatoensis TAKaHASHI (Fagus-Typus) …................. $0.5 \%$

Tricolporopoll. sp. a …................................................................. 1.5\%

7) Periporopoll. asiaticus TAKAHASHI (Liquidambar) ............................... $3 \%$

8) Sporen $=7.5 \%$

? Microreticulatispor. sp. …........................................................... $0.5 \%$

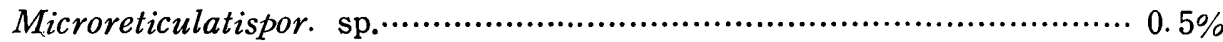

Corrugatispor. haradae TAKAHASHI (Osmundaceae) ….......................... $2 \%$

Verrucatospor. sp. ….............................................................. $0.5 \%$

Gleicheniidites triangularis TaKaHashi n. sp. (cf. Gleicheniaceae) …..... 4\%

Waku (B):

1) Ungeflügelte Koniferen-Pollen $=7 \%$

Inaperturopoll. pseudodubius TAKAHASHI.......................................6.5\%

Sequoiapoll. ligularis (TAKAHASHI) (Sequoia, Metasequoia u. a.) …....... $0.5 \%$

2) Geflügelte Koniferen-Pollen $=1 \%$

Pityospor. orientalis TaKaHaSHI (Pinus-Typus) …............................ 0.5\%

Pityospor. pinoides Takahashi (Pinus-Typus) ….............................. $0.5 \%$

3) Dreieck- und Vieleckpollen $=67.5 \%$

Betulaepoll. rarus TaKaHASHI n. sp. ......................................... $0.5 \%$

Triporopoll. shimensis TaKaHASH (cf. Betulaceae) …..............................

Subtriporopoll. kyushuensis TAKAHASHI (Carya ?) ….............................. 2\%

Subtriporopoll. consimilis TaKaHASHI n. sp. …....................................

Alnipoll. eminens (TAKAHASHI) (Alnus) ….....................................43.5\%

Polyporopoll. grandis TaKaHashi (Ulmaceae ?) …............................. 1.5\%

Polyporopoll. similaris TAKaHASHI (Juglandaceae?) ............................. $5 \%$

Ulmipoll. undulosus WoLfF (Ulmus u. Zelkova) ................................. 3\%

Polyporopoll. sp. ...................................................................... 0.5\%

Tiliaepoll. tiliaceus (TAKAHASHI) …........................................... 8.5\%

Porocolpopoll. sp. …............................................................... $0.5 \%$

4) Tricolpaten -Pollen $=3.5 \%$

Tricolpopoll. umiensis TaKaHaSHI (Cupuliferae) ….......................... $0.5 \%$

Tricolpopoll. meinohamensis TAKAHASHX rotundus TAKAHASHI................ $1 \%$

Tricolpopoll. reticulatus TAKAHASHI (Salix, Platanus u. a.) …….......... $5 \%$

Tricolpopoll. microreticulatus TAKAHASHI (Salix, Platanus u. a.) ).........0.5\%

27: Monocolpopoll. pflugii TakaHashI

28: Monocolpopoll. sp.

29: Tricolporopoll. incertus $\mathrm{T}_{\mathrm{AKAH}} \mathrm{ASHI}$

30: Tricolporopoll. minor $\mathrm{T}_{\text {aKaHashi }}$

31: Tricolporopoll. nagatoensis TAKaHASH

32: Tricolporopoll. microreticulatus THомson \& PFLUG

33: Tricolporopoll. sp. a

34: Tricolporopoll. sp. b
35: Tricolporopoll. sp. c

36: Periporopoll. asiaticus $\mathrm{T}_{\mathrm{AKAHASHI}}$

37: Corrugatispor. haradae TAKAHASHI

38: Gleicheniidites triangularis TAKAHASHI

39: ? Microreticulatispor. sp.

40: Microreticulatispor. sp.

41: Laevigatospor. dehiscens $\mathrm{T}_{\mathrm{AKAHASHI}}$

42: Laevigatospor. sp.

43: Verrucatospor. sp. 
5) Monocolpaten-Pollen $=1.5 \%$

Monocolpopoll. kyushuensis TAKAHASHI (Palmae, Ginkgoinae) ...................1\%

Monocolpopoll. sp. ............................................................. $0.5 \%$

6) Tricolporaten -Pollen $=12 \%$

Tricolporopoll. microreticulatus THomson \& PfLUg …........................ 7.5\%

Tricolporopoll. sp. a …....................................................... $0.5 \%$

Tricolporopoll. sp. b …........................................................... $0.5 \%$

Tricolporopoll. sp. c …......................................................... $0.5 \%$

Ilexpoll. claviger (TAKaHASHI) (Aquifoliaceae) …............................. 0.5\%

Ilexpoll. tertiarius (TaKaHASHI) (cf. Aquifoliaceae) ….......................... 2.5\%

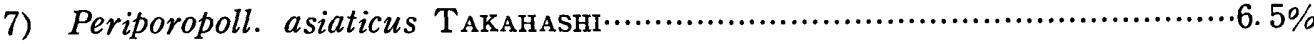

8) Sporen $=1 \%$

Corrugatispor. haradae TAKAHASHI (Osmundaceae) …......................... $0.5 \%$

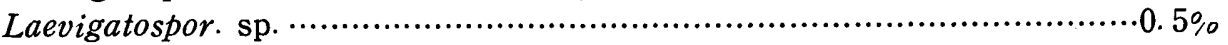

Kiwado:

1) Ungeflügelter Koniferen-Pollen $=5 \%$

Inaperturopoll. pseudodubius T TKAHASHI ….....................................5\%

2) Dreieck- und Vieleckpollen $=89.5 \%$

Triporopoll. shimensis TaKaHASHI (cf. Betulaceae) ….................... $0.5 \%$

Alnipoll. eminens (TAKAHASHI) …........................................ 83.5\%

Polyporopoll. grandis TaKAHASHI (Ulmaceae) …................................ 1\%

Ulmipoll. undulosus WolfF (Ulmus u. Zelkova) …............................. $5 \%$

Tiliaepoll. tiliace us (TAKAHASHI) .............................................. $3 \%$

3) Tricolpaten -Pollen $=2 \%$

Tricolpopoll. ditis Takahashi (Cupuliferae) …................................ 0.5\%

Tricolpopoll. reticulatus TAKAHASHI (Salix, Platanus u. a.) .................. 1\%

Tricolpopoll. striatellus TAKAHASHI …........................................ $0.5 \%$

4) Monocolpaten-Pollen $=1.5 \%$

Monocolpopoll. kyushuensis TaKahaSHI (Palmae, Ginkgoinae) ……............ 1\%

Monocolpopoll. pflugii TaKaHashi (Palmae ?) …................................ 0.5\%

5) Tricolporaten -Pollen $=0.5 \%$

Tricolporopoll. minor TaKaHASHI (cf. Cyrillaceae) …........................ 0.5\%

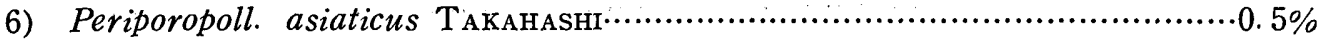

7) Ericipites sp. (Ericaceae) …...................................................... $0.5 \%$

8) Spore $=0.5 \%$

Laevigatospor. dehiscens TAKahaSHI (Polypodiaceae) …................... 0.5\%

Der Verfasser kann die Hioki-Pollengruppe auf Grund ihrer oben erwähnten Eigentümlichkeiten mit dem Sasebo-Pollenbild altersmäßig vergleichen (s. Tabelle 2). Die Ashiya-Schichtengruppe zeigt ebenfalls das Sasebo-Pollenbild. Unter Berücksichtigung der Befunde der Mollusken- und Foraminiferenreste kann er die HiokiSchichtengruppe mit der Ashiya-Schichtengruppe vergleichen. 
Tabelle 2. Stratigraphische Zusammenstellung der oligozänen und untermiozänen Schichten von Nordkyushu und Westhonshu.

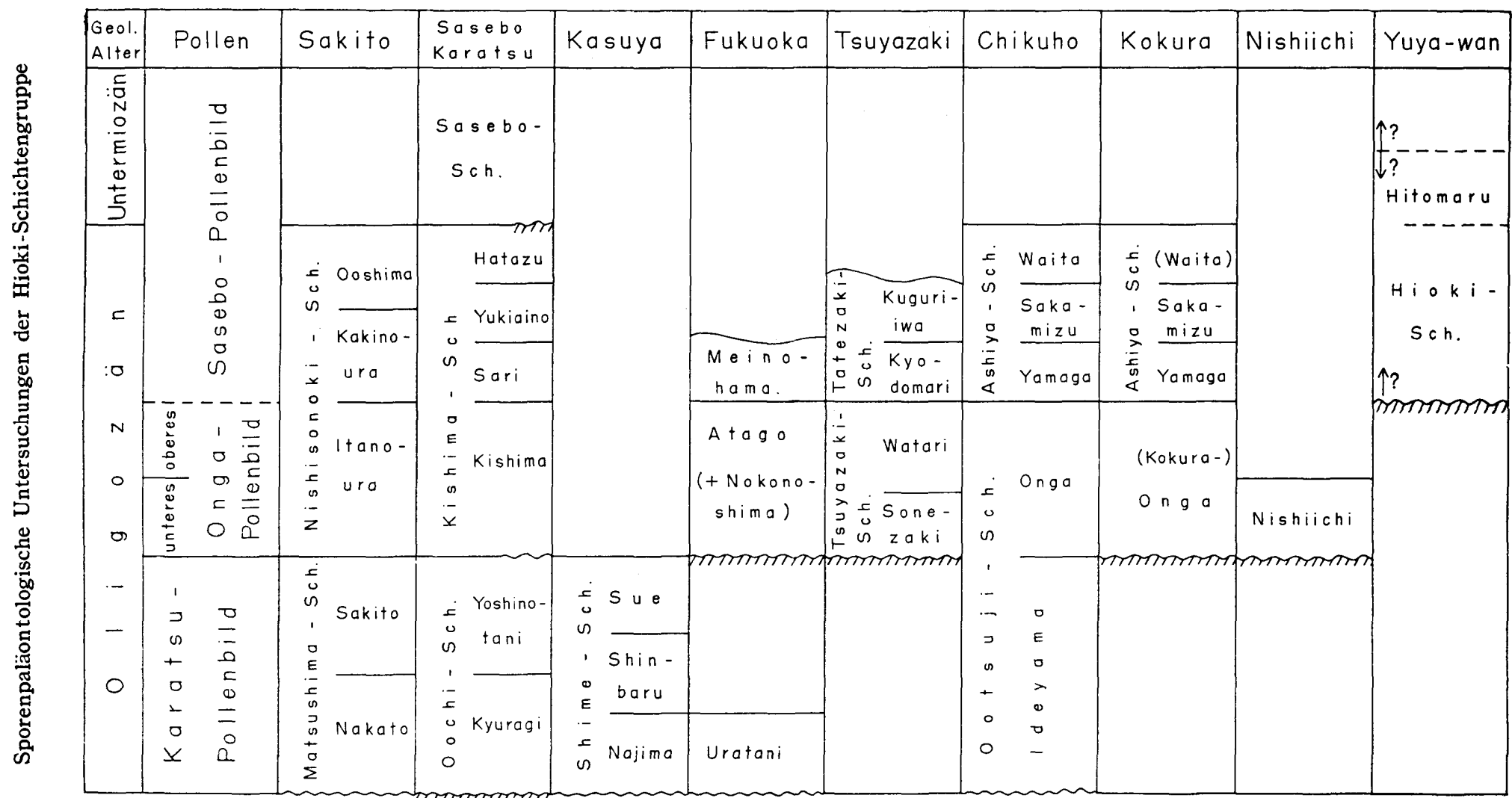

Solche Grenzen zwischen den Bildern und Schichten, die sich noch etwas nach oben oder unten verschieben können, sind als unterbrochene Linie eingezeichnet. 


\title{
Beschreibung der neuen Sporomorphae
}

\author{
Sporites \\ Triletes \\ Formgattung: Gleicheniidites (Ross) Delcourt \& Sprumont, 1955.
}

Gleicheniidites triangularis $\mathrm{n}$. $\mathrm{sp}$.

Taf. 20, Fig. 1-3.

Diagn ose : $28-38.4 \mu$ groß. Konkave Äquatorkontur des Exospors. Exospor chagrenat. End- und Ektexospor dünn, sehr eng vereinigt. Y-Leisten sind schmal, verlaufen geradlinig und erreichen den Äquator. Torus (?).

Holotypus: Ca. $33 \mu$ groß; Taf. 20, Fig. 2; Präparat GK-V 3005.

Locus typicus: Waku (A), Houhoku-machi, Toyoura-gun, Provinz Yamaguchi.

Stratigraphisches Verhalten: Diese Art wurde bisher in den Sakaigawa-Schichten der Hioki-Schichtengruppe und im oberen Matsuurasanjaku-Flöz der Yunoki-Schichten der Sasebo-Schichtengruppe in der Sumitomo-Senryu-Grube, Provinz Nagasaki gefunden.

Beziehungen: Die betreffende neue Spezies ist der skandinavischen oberkretazeischen Art, Gleicheniidites senonicus Ross (1949, Taf. 1, Fig. 3), morphologisch ähnlich. Die Exine der letzteren ist glatt. G. E. Rouse (1957) hat eine ähnliche Form als Cibotiumsporites concavus Rouse (1957, S. 354, Taf. 1, Fig. 36, 37) aus den oberkretazeischen Schichten von Westkanada beschrieben und abgebildet. Sie hat einen deutlichen Torus und ist größer als die betreffende japanische Spezies.

Botanische Zugehörigkeit: Vielleicht Gleicheniaceae.

Formgattung: Microreticulatisporites (Knox) Potoní \& KRemp, 1955.

? Microreticulatisporites $\mathrm{sp}$.

Taf. 20, Fig. 4.

Die vorliegende Art ist ca. $37 \mu$ groß. Äquatorkontur rundlich. Y-Marke undeutlich. Netz ca. $3 \mu$ breit und vieleckig.

Diese Form fand sich in den Sakaigawa-Schichten der Hioki-Schichtengruppe von Waku (A), Houhoku-machi, Toyoura-gun, Provinz Yamaguchi. Sie ist der westjapanischen miozänen Art, Microreticulatisporites (al. Reticulatispor.) saseboensis (TaKahashi) (1961, S. 282-283, Taf. 15, Fig. 1-3) ähnlich.

Die botanische Zugehörigkeit ist fraglich.

\section{Microreticulatisporites sp.}

Taf. 20, Fig. 5 .

Die vorliegende Spezies ist ca. $32 \mu$ groB. Äquatorkontur ursprünglich kreisförmig. Y-Leisten strichförmig schmal, den Äquator erreichend(?), ohne Aufspaltung. Netz rundlich, $1 \mu \pm$ breit.

Sie wurde in den Sakaigawa-Shichten der Hioki-Schichtengruppe von Waku (A), 
Houhoku-machi, Toyoura-gun, Provinz Yamaguchi gefunden. Sie ist der eozänen Spezies, Microreticulatispor. (al. Reticulatispor.) pusillus Takahashi (1962, S. 10-11, Taf. 1, Fig. 5-8), ähnlich.

Die botanische Zugehörigkeit ist fraglich.

\author{
Monoletes \\ Formgattung: Laevigatosporites (IBRAHIM) Potonié \& Kremp, 1956.
}

Laevigatosporites sp.

Taf. 20, Fig. 6 .

Die vorliegende Spezies ist $74.4 \mu$ groß. Figura ellipsoidisch oder bohnenförmig(?). Exospor sehr dünn, sekundär verfaltet, glatt.

Sie fand sich in den Sakaigawa-Schichten der Hioki-Schichtengruppe von Waku (B), Houhoku-machi, Toyoura-gun, Provinz Yamaguchi. Sie ist der westjapanischen miozänen Spezies, Laevigatosporites gigantiformis TAKaHashi (1961, S. 289, Taf. 16, Fig. 3), ähnlich, aber der Verfasser kann sie hier nur als Laevigatospor. sp. beschreiben und abbilden, da sie sekundär stark verfaltet ist.

Sie gehört vielleicht $z u$ den Polypodiaceen.

\title{
Pollenites
}

Brevaxones

Postnormapolles

Formgattung: Betulaepollenites R. Potonié, 1934.

Betulaepollenites rarus n. sp.

Taf. 22, Fig. 1, 2.

Diagnose: 24-26 $\mu$ groß. Kontur rundlich-dreieckig. Exine mit Atrium (?), aber ohne Anulus und Tumeszenz, stets mit Plicae, die als kräftige Quellfalten der gesamten für diese Gruppe bezeichnend sind. Exine bis $1.5 \mu$ dick, chagrenat. Der Diameter der Poren ist größer, der Porenkanal nicht tief.

Hol otypus: $24 \mu$ groß; Taf. 22, Fig. 2; Präparat GK-V 3017. guchi.

Locus typicus: Waku(B), Houhoku-machi, Toyoura-gun, Provinz Yama-

Stratigraphisches Verhalten: Diese neue Spezies wurde bisher nur in den Sakaigawa-Schichten der Hioki-Schichtengruppe (oberes Oligozän) von Waku gefunden.

Bezi e hungen: Die rarus-Form ist den mitteleuropäischen tertiären Spezies, Triatriopoll. pseudosporites Pflug (Thomson \& Pflug, 1953, S. 78, Taf. 7, Fig. 32-35) und Triatriopoll. excelsus (R. Pot) Pflug subsp. turgidus Pflug (Thomson \& Pflug, 1953, Taf. 7, Fig. 36-46), ähnlich. Beide mitteleuropäische Spezies haben einen Anulus. Die rarus-Form ist auch der westjapanischen Art, Betulaepoll. (al. Triatriopoll). sp. (mit Plicae) (K. TakahashI, 1961, Taf. 19, Fig. 2, 3), ähnlich. 
Die letztere ist nicht rundlich, sondern deutlich dreieckig.

Botanische Zugehörigkeit: Vielleicht Betulaceae.

Formgattung: Subtriporopollenites Thомson \& Pflug, 1953.

Subtriporopollenites consimilis n. sp.

Taf. 22, Fig. 12, 13.

Diagnose: 44.7-50 $\mu$ groß. Kontur kreisrund. Poren rundlich bis etwas elliptisch, normalerweise alle subäquatorial. Exine verhältnismäßig dick $(1.6$ bis $2.2 \mu$ dick), chagrenat, sekundär verfaltet.

Holotypus: Ca. $47 \mu$ groB; Taf. 22, Fig. 12; Präparat GK-V 3017.

Locus ty picus: Waku(B), Houhoku-machi, Toyoura-gun, Provinz Yamaguchi.

Stratigraphisches Verhalten: Diese neue Spezies wurde bisher nur in den Sakaigawa-Schichten der Hioki-Schichtengruppe (oberes Oligozän) von Waku und in den Hitomaru-Schichten gefunden.

Be z i e h ungen: Die consimilis-Form ist der mitteleuropäischen tertiären Spezies, Subtriporopoll. simplex(R. Pот. \& Ven.) Thomson \& Pflug subsp. simplex (R. Pot. \& Ven.) Thomson \& Pflug (1953, S. 86, Taf. 9, Fig. 64-73), morphologisch ähnlich. Der Exinenindex der letzteren liegt unter 0.1, aber bei der ersteren nicht immer unter 0.1. Die erstere ist auch der japanischen Spezies, Subtriporopoll. kyushuensis Takahashi (1961, S. 305-306, Taf. 20, Fig. 35-37; Taf. 21, Fig. 1-10), ähnlich. Die kyushuensis-Form ist kleiner als die consimilis-Form. Die Exine der letzteren ist zweimal dicker als die der ersteren.

Botanische Zugehörigkeit: Juglandaceae.

Formgattung: Polyporopollenites PfLug, 1953.

\section{Polyporopollenites sp.}

Taf. 22, Fig. 8 .

Diese Art ist $36.7 \mu$ groß. Sechsporig. Kontur sechseckig mit geraden Seiten. Poren meist alle äquatorial. Exine in Seitenmitte $0.8 \mu$ dick, mit schwachem Anulus von etwas tropfenförmigem Querschnitt. Intrapunktate oder chagrenate Skulptur.

Die betreffende Art fand sich nur in den Sakaigawa-Schichten der Hioki-Schichtengruppe von Waku (B), Houhoku-machi, Toyoura-gun, Provinz Yamaguchi.

Sie ist den anderen japanischen Arten, Polyporopoll. similaris Takahashi (1961, S. 309, Taf. 21, Fig. 25-30) und Polyporopoll. polyceras TaKahashi (1961, S. 309-310, Taf. 22, Fig. 1-3), ähnlich. Die similaris-Form hat einen deutlichen Anulus von kugeligem bis keulenförmigem Querschnitt. Die polyceras-Form besitzt keinen Anulus und ein schwaches Labrum.

Botanische Zugehörigkeit: Juglandaceae. 
Formgattung: Porocolpopollenites PfLug, 1953.

Vestibuloidae

Porocolpopollenites sp.

Taf. 22, Fig. 14.

Die vorliegende Art ist ca. $34 \mu$ groß. Kontur dreieckig mit schwach konkaven Seiten. Colpen deutlich. Mehr oder weniger kräftige rugulate Skulptur, die in Form von Muri $1 \mu$ hoch ist.

Sie fand sich nur in den Sakaigawa-Schichten der Hioki-Schichtengruppe von Waku (B), Houhoku-machi, Toyoura-gun, Provinz Yamaguchi. Diese Form ist der mitteleuropäischen tertiären Spezies, Porocolpopoll. vestibulum (R. Pot.) Pflug (Thomson \& Pflug, 1953, Taf. 11, Fig. 3-23), offenbar ähnlich.

Botanische Zugehörigkeit: Symplocaceae.

\section{Longaxones}

Formgattung: Tricolporopollenites Thomson \& Pflug, 1953.

Tricolporopollenites sp. a

Taf. 21, Fig. 11. 12.

Diese Art ist 32 bis $34.5 \mu$ groß. Kontur rundlich bis breit-elliptisch. Figura kugelig bis breit-ellipsoidisch. Breitenlängenindex 0.85 bis 0.9 . Polkappenkontur halbkugelig bis unterhalbkugelig. Großer(?) und rundlicher Porus, über die Caverna hinausgreifend. Exine dünn, foveolat-oder fossulatartig skulptiert.

Die betreffende Art ist den mitteleuropäischen tertiären Arten, Tricolporopoll. sustmanni Thomson \& Pflug (1953, S. 101, Taf. 12, Fig. 122) und Tricolporopoll. genuinus (R. Poт.) Thomson \& Pflug (1953, S. 105, Taf. 13, Fig. 69-85), offenbar ähnlich. Sie wurde in den Sakaigawa-Schichten der Hioki-Schichtengruppe von Waku (A, B), Houhoku-machi, Toyoura-gun, Provinz Yamaguchi gefunden.

Die botanische Zugehörigkeit ist fraglich.

\section{Tricolporopollenites sp. $\mathrm{b}$}

Taf. 21, Fig. 14.

Die betreffende Spezies ist ca. $36 \mu$ groß. Kontur elliptisch. Figura ellipsoidisch. Breitenlängenindex ca. 0.6. Polkappenkontur halbkugelig bis zugespitzt. Porus rundlich (?). Exine $1.6 \mu$ dick, intrarugulat.

Sie wurde bisher nur in den Sakaigawa-Schichten der Hioki-Schichtengruppe von Waku (B), Houhoku-machi, Toyoura-gun, Provinz Yamaguchi gefunden.

Die botanische Zugehörigkeit ist fraglich.

\section{Tricolporopollenites sp. c}

Taf. 21, Fig. 16. 
Die Art ist $49 \mu$ groß. Kontur elliptisch. Figura ellipsoidisch. Breitenlängenindex ca. 0.5. Polkappenkontur zugespitzt. Porus undeutlich. Exine fossulat oder foveolatartig skulptiert.

Sie wurde in den Sakaigawa-Schichten der Hioki-Schichtengruppe von Waku (B), Houhoku-machi, Toyoura-gun, Provinz Yamaguchi gefunden. Sie ist der eozänen Art, Tricolporopoll. hoshuyamaensis TAKAHASHI subsp. fossulatus TAKAHASHI (1961, S. 325-326, Taf. 25, Fig. 5-9), sehr ähnlich.

Die botanische Zugehörigkeit ist fraglich.

\section{Literaturverzeichnis}

Болховитина, Н. А. (1961): Ископаемые и современные споры семейства схизейных. Akадемия Hayk СССР, Труды Геологичесkого Ннститута, Выпуск 40, 1-176, Та6. 1-41.

Couper, R. A. (1953): Upper Mesozoic and Cainozoic spores and pollen grains from New Zealand. New Zealand geol. Surv. paleont. Bull., 22, 1-77, pls. 9, textfigs. 3, tab. 3.

- (1954): Plant microfossils from New Zealand No. 1. Trans. Roy. Soc. New Zealand, 81, (4), 479-483, figs. 9, textfig. 1.

Erdtman, G. (1952): Pollen morphology and plant taxonomy, angiosperms. 539, Chronica Botanica Company.

- (1954): An introduction to pollen analysis. 239, Chronica Botanica Company.

IkUse, Masa (1956): Pollen grains of Japan. (in Japanese), 303, pls. 1-76, Hirokawa publishing Co., Tokyo.

IMAMURA, S. und $\mathrm{W}_{\mathrm{ADA}}, \mathrm{K}$. (1956): Über die Hishikai-Schichtengruppe in der Yuyawan Gegend, Prov. Yamaguchi (Abstrakt). (jap.), Jour. geol. Soc. Japan, 62, (730), 390. und (1958): Das von fossiler Flora geurteilte geologische Alter der AshiyaSchichtengruppe in der Yuyawan Gegend, Prov. Yamaguchi (Abstrakt). (jap.), Jour. geol. Soc. Japan, 64, (759), 700.

IмамURA, S. und Окамото, K. (1959): Stratigraphie und Struktur der jung- und alttertiären Schichten in der Yuyawan Gegend, Prov. Yamaguchi (Abstrakt). (jap.), Jour. geol. Soc. Japan, 65, (766), 440-441.

Krutzscr, W. (1957): Sporen und Pollengruppen aus der Oberkreide und dem Tertiär Mitteleuropas und ihre stratigraphische Verteilung. Z. angewandte Geol., Heft 11/12, 509-548, Taf. 1-16, Tab. 1-2.

Mürriger, F. und Pflanzl, G. (1955): Pollenanalytische Datierung einiger hessischer Braunkohlen. Notizbl. hess. L. -Amt Bodenforsch., 83, 71-89.

Oaura, T. (1918): Geologischer Bericht von Ootsu-gun, Prov. Yamaguchi. (jap.), Rep. geol. Survey Japan, (66), 1-48.

- (1919): Uber die tertiären Schichten von Ootsu-gun, Prov. Yamaguchi. (jap.). Jour. Geogr., 31, (364), 238-245.

Окамото, Kazuo (1960): Kleinforaminiferengruppe der tertiären Schichten, bei Yuyawan Gegend, Prov. Yamaguchi (vorläufige Mitteilung). (jap.), Foraminiferen (Yukoochu), 11, 47-53.

- (1961): Cenozoic formations of Tsuno-shima (Island), Hohoku-cho, Toyoura-gun, Yamaguchi Prefectre (in Japanese with English abstract), Jour. geol. Soc. Japan, 67, (791), 476-483.

Окамото, K. und Imamura, S. (1962): Tertiäres System bei Yuyawan, Prov. Yamaguchi, besonders über die "Ashiya"-Schichtengruppe (Abstrakt), (jap.), Jour. geol. Soc. Japan, 68, (802), 413-414.

Prlangu, G. (1956): Das Alter der Braunkohlen des Meissners, der Flöze 2 und 3 des Hirschberges und eines benachbarten Kohlenlagers bei Landenbach. Notizbl. hess. L.-Amt Bodenforsch., 84, 232-244.

Pflug, H. D. (1953): Zur Entstehung und Entwicklung des angiospermiden Pollens in der Erd- 
geschichte. Palaeontographica, B, 95, 60-171.

(1956): Beiträge zur Klimageschichte Islands II. Sporen und Pollen von Tröllatunga (Island) und ihre Stellung $\mathrm{zu}$ den pollenstratigraphischen Mitteleuropas. N. Jb. geol. paläont., Abh. 102, (3), 409-430, Taf. 17-19.

(1959): Beiträge zur Klimageschichte Islands VIII. Sporenbilder aus Island und ihre stratigraphische Deutung. N. Jb. geol. paläont., Abh. 107, (2), 141-172, Taf. 12-16.

Potoní́, R. (1956): Synopsis der Gattungen der Sporae dispersae. I. Teil: Sporites. Beih. geol. $J b .$, (23), 1-103, Taf. 1-11.

(1958): Synopsis der Gattungen der Sporae dispersae. II. Teil: Sporites (Nachträge), Saccites, Aletes, Praecolpates, Polyplicates, Monocolpates. Beih. Geol. Jb., (31), 1-114, Taf. 1-11.

(1960): Sporologie der eozänen Kohle von Kalewa in Burma. Senckenbergiana lethaea, 41, $1 / 6,451-481$, Taf. 1-2.

Ross, N. E. (1949): Investigations of the Senonian of the Kristianstad district, s. Sweden. I. On a Cretaceous pollen and spore bearing clay deposit of Scania. A preliminary report. Bull. Geol. Inst. Univ. Upsala, 34, 25-43, pls. 3.

Rouse, G. (1957): The application of a new nomenclatural approach to upper Cretaceous plant microfossils from western Canada. Can. J. Bot., 35, 349-375, pls. 3.

TAKAHASHI, Kiyoshi (1957): Palynologisch-stratigraphische Untersuchung der tertiären Schichten im Kasuya und Fukuoka Kohlenfeld von Nordkyushu, Japan. Mem. Fac. Sci., Kyushu Univ., 5, (4), 199-221, Taf. 38-39, Abb. 1-6, Tab. 1-3.

(1961): Pollen und Sporen des westjapanischen Alttertiärs und Miozäns (I), (II). Mem. Fac. Sci., Kyushu Univ. 11, (2), 151-255, Tab. 1-15, Abb. 1-40; (3), 279-345, Taf. 13-27.

(1962): Pollenformen aus den eozänen Kohlenflözen von Ishizuchi. Mem. Fac. Sci., Kyushu Univ., 12, (1), 1-26, Tab. 1, Abb. 1-2, Taf. 1-5.

(1963): Pollen und Sporen aus dem Schieferton von Ashiya, Nordkyushu. Jap. Jour. Geol. Geogr.

(1963): Pollenformen aus den Hitomaru-Schichten in der Yuyawan Gegend. Trans. Proc. Palaeont. Soc. Japan. N. S.

Thiergart, F. (1940): Die Mikropaläontologie als Pollenanalyse im Dienst der Braunkohlenforschung. Brennstoff-Geol., Heft 13, 1-82, Taf. 1-14, Tab. 3.

Thomson, P. W. und Pflug, H. D. (1953): Pollen und Sporen des mitteleuropäischen Tertiärs. Palaeontographica, B, 94, 1-138, Taf. 1-15, Abb. 20, Tab. 4.

Traverse, Alfred (1955): Pollen analysis of the Brandon lignite of Vermont. Bureau of Mines, Rept. Invest. 5151, U. S. Dep.t Interior, 1-107, pls. 8-13, text- figs. 1-7.

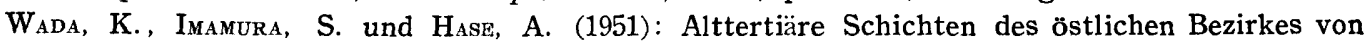
Yuyawan, Ootsu-gun, Prov. Yamaguchi. (jap.), (Abstrakt), Jour. Geol. Soc. Japan, 57, (670), 306.

Wada, K. und Imamura, S. (1952): Alttertiäre Schichten des nordwestlichen Bezirkes von Toyoura-gun, Prov. Yamaguchi (jap.) (Abstrakt). Jour. Geol. Soc. Japan, 58, (682), 309.

Weyland, H. und Krieger, W. (1953): Die Sporen und Pollen der Aachener Kreide und ihre Bedeutung für die Charaktersierung des mittleren Senons. Palaeontographica, B, 95, 3052, Taf. 6-13, Abb. 4.

Weyuand, H. und Pflua, H. D. (1957): Die Pflanzenreste der pliozänen Braunkohle von Ptolemais in Nordgriechenland I. Palaeontographica, B, 102, 96-109, Taf. 21-22.

Weyland, H. und TaKaHashr, K. (1961): Pflanzenreste aus der Braunkohlengrube "Herman" bei Heerlen, Holländisch Limburg. Palaeotographica, B, 109, 93-107, Taf. 42-44.

Wodehouse, R. P. (1933): Tertiary Pollen. II. The oil shales of the Eocene Green River formation. Bull. Torr. Bot. Club, 60, 479-524, text-figs. 56.

(1935): Pollen grains. 574, McGraw-Hill book Company, New York and London.

Z ARLINSKAJA, E. D. (1957): Stratigraphic significance of pollen grains of gymnosperms of the Cenozoic deposits of the Irtysh basin and of the northern Aral basin. (in catalog of fossil spores and pollen, 4), Acad. Sci. USSR, works Geol. Inst. Contr., 6, 1-184, pls. 17. 
Kiyoshi Takahashi

Sporenpaläontologische Untersuchungen

der Hioki-Schichtengruppe von Waku und Kiwado

\section{Tafeln 20-22}


Tafel 20 


\title{
Erklärung zu Tafel 20
}

\author{
(Ca. 832 fach vergr.)
}

Fig. 1-3. Gleicheniidites triangularis n. sp.

Waku (A), Houhoku-machi, Toyoura-gun, Provinz Yamaguchi. Fig. 1: Präparat GK-V 3007; Fig. 2: Präparat GK-V 3005, Holotypus; Fig. 3: Präparat GK-V 3006.

Fig. 4. ? Microreticulatispor. sp.

Waku (A), Houhoku-machi, Toyoura-gun, Provinz Yamaguchi. Präparat GK-V 3001.

Fig. 5. Microreticulatispor. sp.

Waku (A), Houhoku-machi, Toyoura-gun, Provinz Yamaguchi. Präparat GK-V 3004.

Fig. 6. Laevigatospor. sp.

Waku (B), Houhoku-machi, Toyoura-gun, Provinz Yamaguchi. Präparat GK-V 3016.

Fig. 7-10. Corrugatispor. haradae TAKAHASHI

Fig. 7, 8, 10: Waku (A), Houhoku-machi, Toyoura-gun, Provinz Yamaguchi. Fig. 9: Waku (B), Houhoku-machi, Toyoura-gun, Provinz Yamaguchi; Präparat GK-V 3018. Fig. 7, 8: Präparat GK-V 3004; Fig. 10: Präparat GK-V 3005 . 

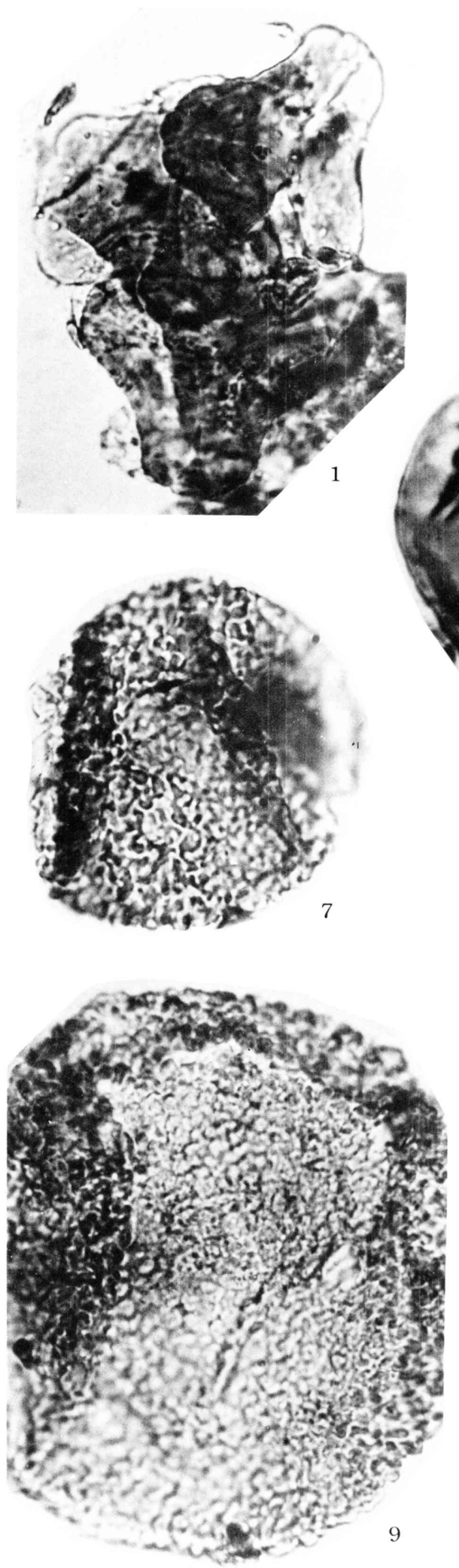
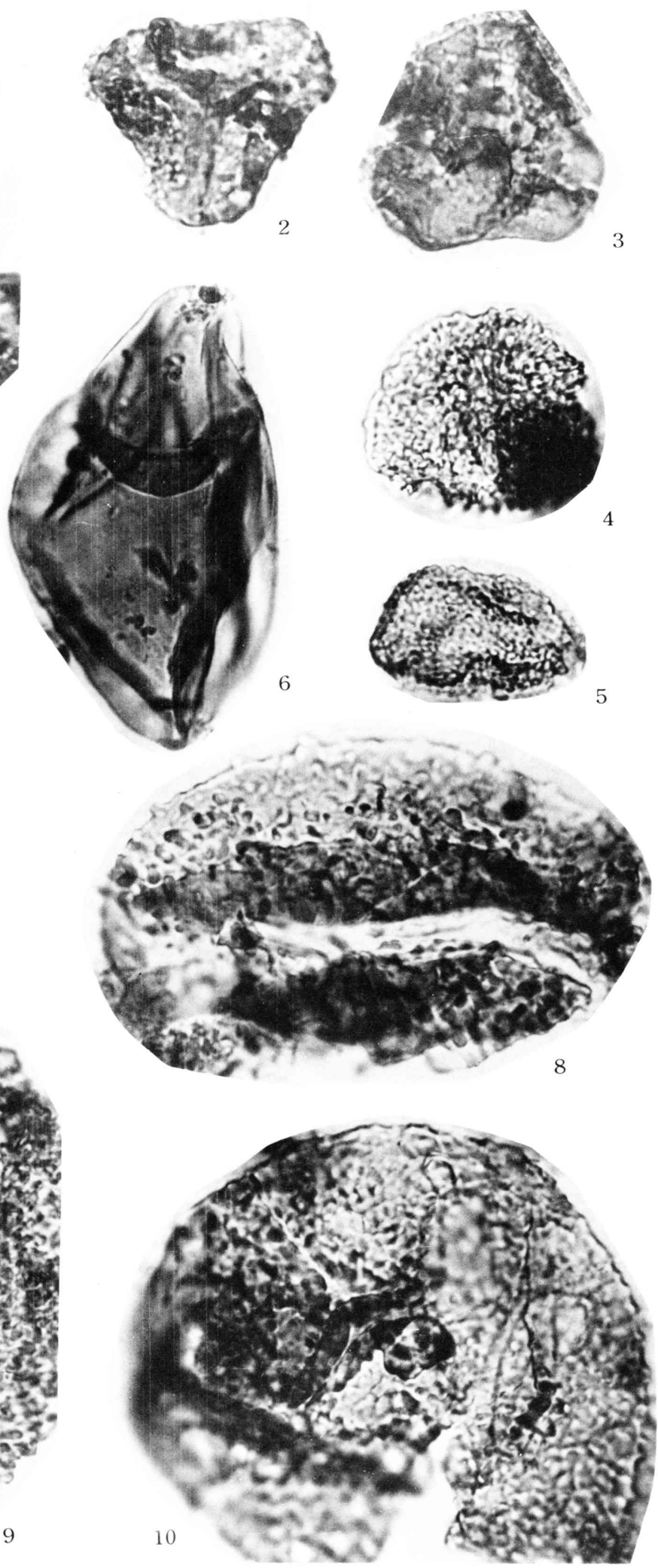

K. TAKahashi: Sporenpaläontologische Untersuchungen der Hioki-Schichtengruppe 
Tafel 21 


\title{
Erklärung zu Tafel 21
}

\author{
(Ca. 832 fach vergr.)
}

Fig. 1. Pityospor. pinoides Takahnsh

Waku (B), Houhoku-machi, Toyoura-gun, Provinz Yamaguchi; Präparat GK-V 3020.

Fig. 2. Inaperturopoll. pseudodubius TAKAHASHI

Waku (A), Houhoku-machi, Toyoura-gun, Provinz Yamaguchi; Präparat GK-V 3005.

Fig. 3. Monocolpopoll. pflugii $\mathrm{T}_{\mathrm{AK} \text { AHashi }}$ Kiwado, Heki-mura, Ootsu-gun, Provinz Yamaguchi; Präparat GK-V 3031.

Fig. 4. ? Monocolpopoll. intrabaculatus TAKAHASHI

Waku (A), Houhoku-machi, Toyoura-gun, Provinz Yamaguchi; Präparat GK-V 3004.

Fig. 5. Tricolpopoll. umiensis TakahashI

Waku (B), Houhoku-machi, Toyoura-gun, Provinz Yamaguchi; Präparat GK-V 3016.

Fig. 6. Tricolpopoll. vulgaris TаканаSH

Waku (B), Houhoku-machi, Toyoura-gun, Provinz Yamaguchi; Präparat GK-V 3005.

Fig. 7. Tricolpopoll. chikushiensis $\mathrm{T}_{\mathrm{AK} A H A S H}$ grandiformis $\mathrm{T}_{\mathrm{AK} A H A S H I}$

Waku (A), Houhoku-machi, Toyoura-gun, Provinz Yamaguchi; Präparat GK-V 3005.

Fig. 8. Tricolpopoll. striatellus TAKaHaSHI

Waku (A), Houhoku-machi, Toyoura-gun, Provinz Yamaguchi; Präparat GK-V 3005.

Fig. 9, 10. Tricolpopoll. reticulatus TAKaHasHI $_{\text {A }}$

Fig. 9: Waku (A), Houhoku-machi, Toyoura-gun, Provinz Yamaguchi; Präparat GK-V 3002; Fig. 10: Kiwado, Heki-mura, Ootsu-gun, Provinz Yamaguchi; Präparat GK-V 3031.

Fig. 11, 12. Tricolporopoll. sp. a

Waku (A), Houhoku-machi, Toyoura-gun, Provinz Yamaguchi. Fig. 11: Präparat GK-V 3002; Fig. 12: Präparat GK-V 3001.

Fig. 13. Tricolporopoll. minor TAKaHASHI

Kiwado, Heki-mura, Ootsu-gun, Provinz Yamaguchi; Präpart GK-V 3031.

Fig. 14. Tricolporopoll. sp. b

Waku (B), Houhoku-machi, Toyoura-gun, Provinz Yamaguchi; Präparat GK-V 3016

Fig. 15. Tricolporopoll. hitomaruensis $\mathrm{T}_{\mathrm{AKAHASHI}}$

Waku (A), Houhoku-machi, Toyoura-gun, Provinz Yamaguchi; Präparat GK-V 3002.

Fig. 16. Tricolporopoll. sp. c

Waku (B), Houhoku-machi, Toyoura-gun, Provinz Yamaguchi; Präparat GK-V 3021.

Fig. 17. Tricolporopoll. microreticulatus Thomson \& Pflug

Waku (B), Houhoku-machi, Toyoura-gun, Provinz Yamaguchi; Präparat GK-V 3017.

Fig. 18. Ilexpoll. tertiarius ( $\mathrm{T}_{\mathrm{AKAH}} \mathrm{ASH}$ )

Waku (B), Houhoku-machi, Toyoura-gun, Provinz Yamaguchi; Präparat GK-V 3021.

Fig. 19-27. Tiliaepoll. tiliaceus ('Tаканаян)

Fig. 19-26: Waku (B), Houhoku-machi, Toyoura-gun, Provinz Yamaguchi: Fig. 19-22: Präparat GK-V 3017; Fig. 23: Präparat GK-V 3016; Fig. 24: Präparat GK-V 3018; Fig. 25, 26: Präparat GK-V 3020; Fig. 27; Waku (A), Houhoku-machi, Toyoura-gun, Provinz Yamaguchi; Präparat GK-V3003. 

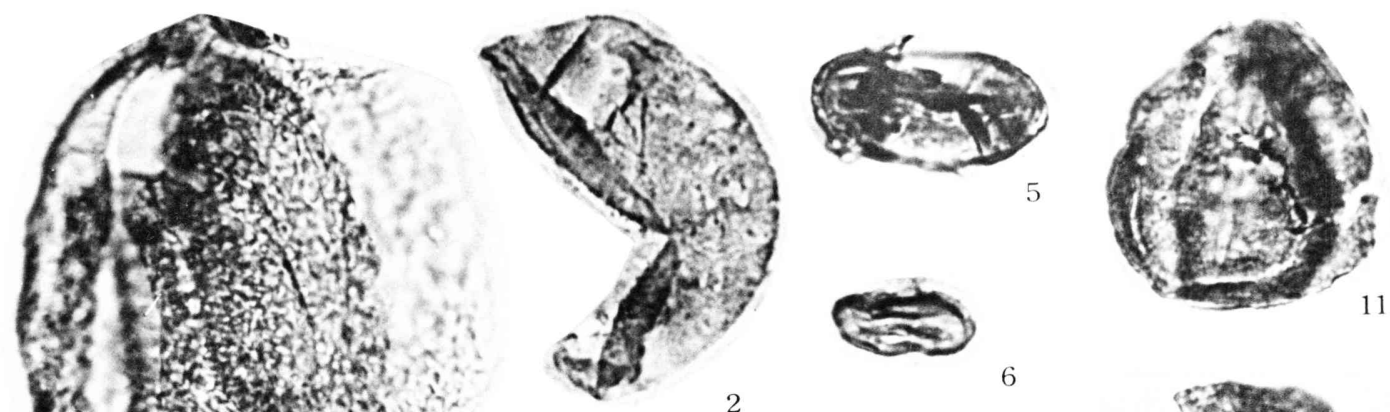

$\operatorname{lin}^{2}, 1,1, \frac{1}{2}$
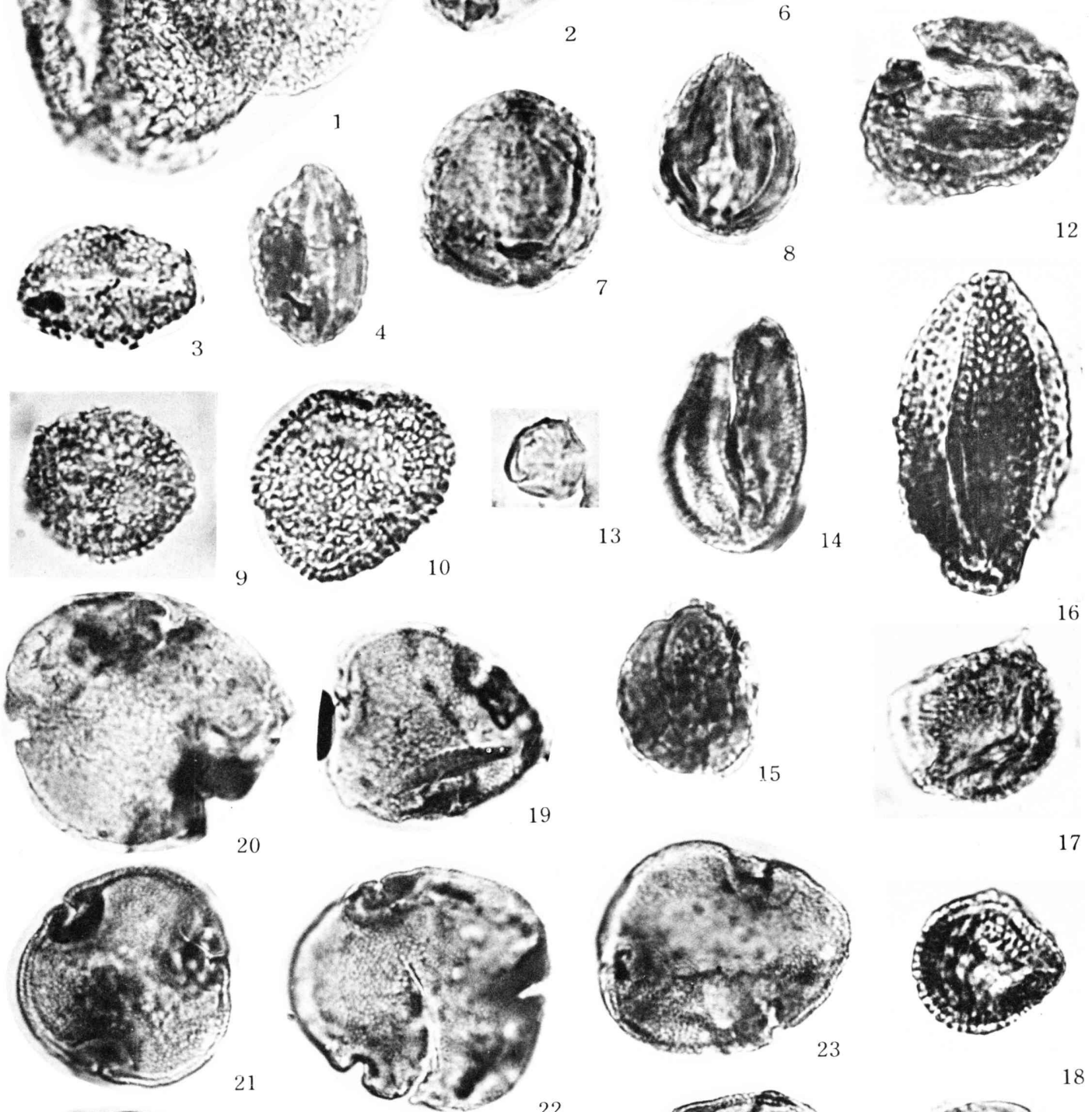

17
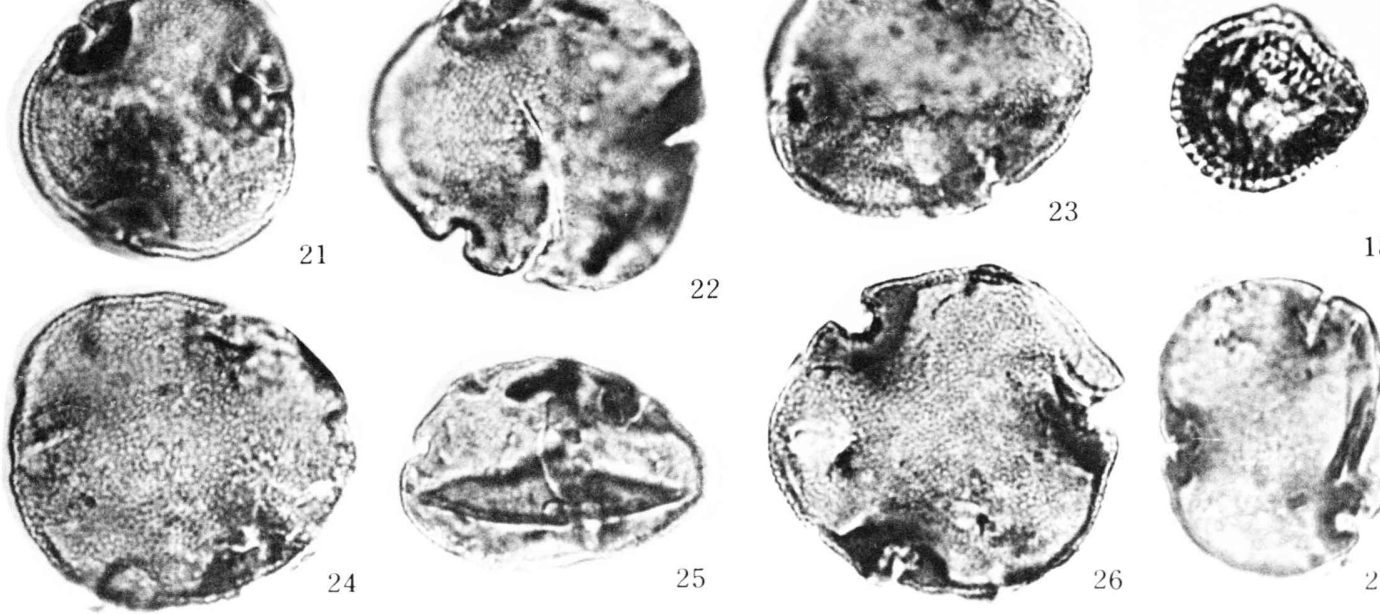

18

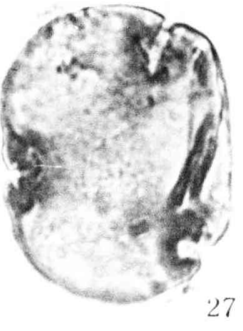

K. Takahashi: Sporenpaläontologische Untersuchungen der Hioki-Schichtengruppe 
Tafel 22 


\title{
Erklärung zu Tafel 22
}

\author{
(Ca. 832 fach vergr.)
}

Fig. 1, 2. Betulacpoll. rarus n. sp.

Fig. 1: Waku (A), Houhoku-machi, Toyoura-gun, Provinz Yamaguchi; Präparat GK-V 3001; Fig. 2: Waku (B), Houhoku-machi, Toyoura-gun, Provinz Yamaguchi; Präparat GK-V 3017.

Fig. 3. Triporopoll. shimensis TakaHasiI

Waku (A), Houhoku-machi, Toyoura-gun, Provinz Yamaguchi; Präparat GK-V 3005.

Fig. 4-7. Alnipoll. eminens (Takahashi)

Fig. 4, 5: Waku (A), Houhoku-machi, Toyoura-gun, Provinz Yamaguchi; Fig. 6, 7: Kiwado, Heki-mura, Ootsu-gun, Provinz Yamaguchi. Fig. 4: Präparat GK-V 3005; Fig. 5: Präparat GK-V 3004; Fig. 6, 7: Präparat GK-V 3031.

Fig. 8. Polyporopoll. sp.

Waku (B), Houhoku-machi, Toyoura-gun, Provinz Yamaguchi; Präparat GK-V 3019.

Fig. 9, 10. Polyporopoll. similaris TakahasiI

Fig. 9: Waku (A), Houhoku-machi, Toyoura-gun, Provinz Yamaguchi Präparat GK-V 3006; Fig. 10: Waku (B), Houhoku-machi, Toyoura-gun, Provinz Yamaguchi; Präparat GK-V 3020.

Fig. 11. Polyporopoll. grandis TAKaHASH

Waku (A), Houhoku-machi, Toyoura-gun, Provinz Yamaguchi; Präparat GK-V 3001.

Fig. 12, 13. Subtriporopoll. consimilis n. sp.

Waku (B), Houhoku-machi, Toyoura-gun, Provinz Yamaguchi; Fig. 12: Präparat GK-V 3017; Holotypus; Fig. 13; Präparat GK-V 3016.

Fig. 14. Porocolpopoll. sp.

Waku (B), Houhoku-machi, Toyoura-gun, Provinz Yamaguchi; Präparat GK-V 3016.

Fig. 15, 16. Ulmipoll. undulosus WoLfF

Fig. 15: Waku (B), Houhoku-machi, Toyoura-gun, Provinz Yamaguchi; Präparat GK-V 3016; Fig. 16: Waku (A), Houhoku-machi, Toyoura-gun, Provinz Yamaguchi; Präparat GK-V 3005.

Fig. 17, 18. Periporopoll. asiaticus TAKAHASHI

Fig. 17: Kiwado, Heki-mura, Ootsu-gun Provinz Yamaguchi; Präprat GKV 3031; Fig. 18: Waku (B), Houhoku-machi, Toyoura-gun, Provinz Yamaguchi; Präparat GK-V 3016.

Fig. 19. Ericipites sp.

Kiwado, Heki-mura, Ootsu-gun, Provinz Yamaguchi; Präparat GK-V 3031.

Fig. 20. Unbestimmbarer Rest. 

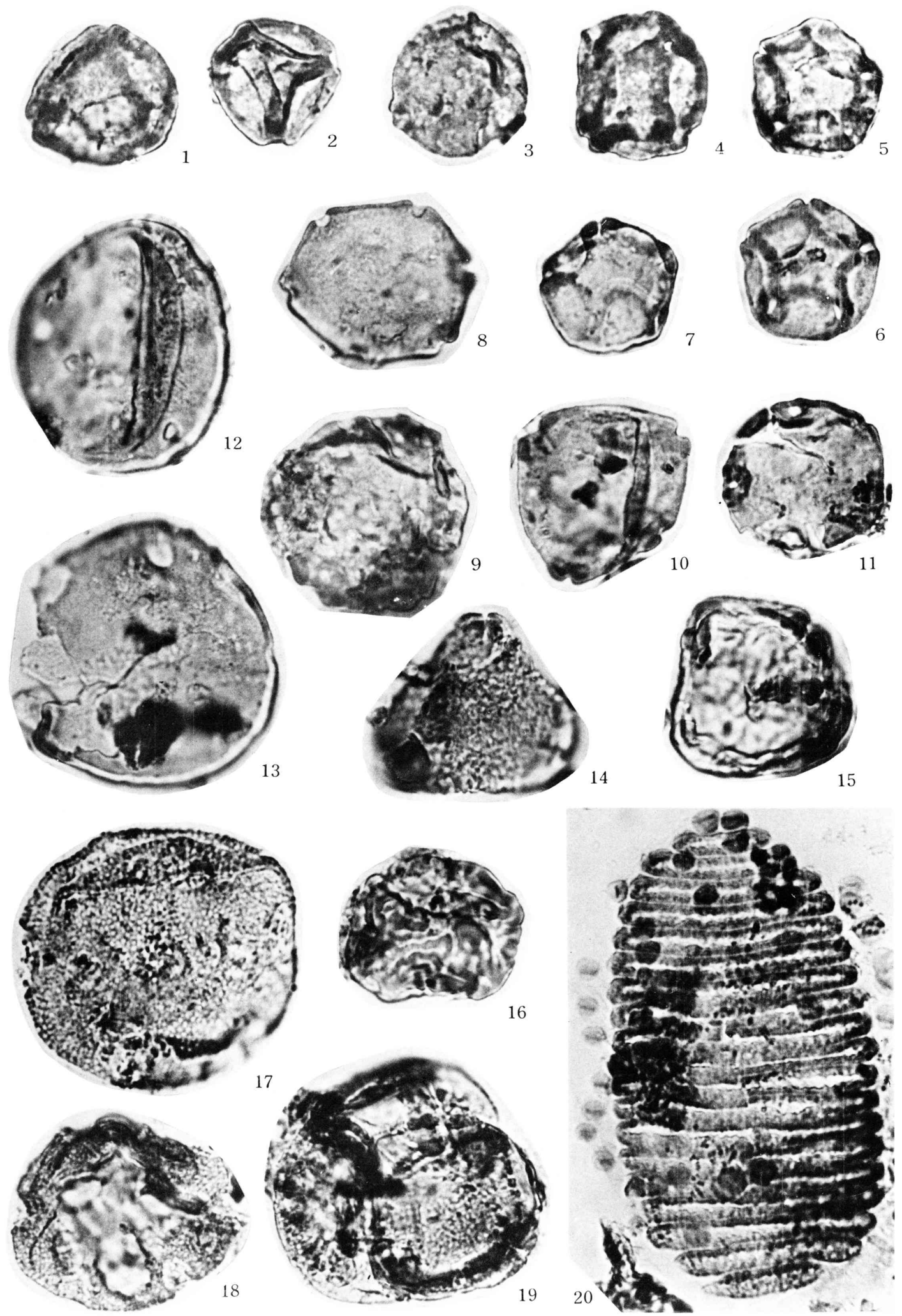

K. Tаканаsнi: Sporenpaläontologische Untersuchungen der Hioki-Schichtengruppe 